\title{
Rabin-Karp Elaboration in Comparing Pattern Based on Hash Data
}

\author{
Andysah Putera Utama Siahaan ${ }^{1,2}$ \\ ${ }^{1}$ Faculty of Computer Science, Universitas Pembangunan Panca Budi, \\ Medan, Indonesia \\ ${ }^{2}$ School of Communication and Computer Engineering, \\ Universiti Malysia Perlis, Kangar, Malaysia \\ andiesiahaan@gmail.com
}

\begin{abstract}
The Rabin-Karp algorithm is known as the algorithm used to analyze a document based on a hash value. This algorithm is not for testing the image pattern. However, hash value can be occupied to compare the images patterns. K-Gram is a hash value-forming component. It determines the relationship between adjacent pixels in each image. Adjustment of base and modulo values affects the image similarity level. The samples used are flower images consisting of train and test data. Each sample will be analyzed by this method and compared with the test image. The image tested is the grayscale image. This analysis uses gram $=2$. The lower the value used, the higher the accuracy is generated. Modulo adjustment also affects the hash value. The combination of the three values is the important factor in image testing. The creation of a feature value against the resulting hash values aims to compare the proximity between two images. The feature extraction function will calculate the distance between the two hashes using Euclidean. The comparison applies to two similar-sized images. The smaller the feature value, the more similar the two images are. This approach produces similar levels according to the processed parameters.
\end{abstract}

Keywords: Image Recognition, Similarity, Rabin-Karp, Pattern

\section{Introduction}

A picture has a thousand meanings. Images are often performed to describe an event or activity in the digital world [10][11]. Using images, this can replace a thousand words. Searching for data by an image is a difficult thing to do because the image has a considerable amount of pixel data. It is different when searching for a string. The string only has a few characters long [1][2][3].

There are many algorithms for image matching [9]. In general, in digital image processing, image matching is done with the help of artificial neural networks. The image will be divided into two types, training and testing. Rabin-Karp is a string matching method that utilizes the word pieces in each string [7][8]. This algorithm has hash value to determine the feature value of each piece of the word [4][5][12]. This algorithm can be developed or tested using an image as a test medium. In the present study, it will be attempted to test Rabin-Karp's method of image pattern. The results of the calculation are expected to determine how similar the image test with the image in the training data.

Received (November 5, 2017), Review Result (January 18, 2018), Accepted (February 1, 2018) 


\section{Related Work}

Ashish Prosad Gope and Rabbi Narayan Behera had research in 2014. Their research examined the relationship of the Rabin-Karp method with Deoxyribose Nucleic Acid (DNA). DNA has its own rules and sequences in the order. This arrangement is to determine the nucleotides present in DNA. Each sequence has a specific pattern for determining human genes. Their research is a field of bioinformatics research. This study combines computer science with biology. The purpose of this study was to find suitable algorithms to determine patterns that exist in DNA. This research tested several matching algorithms. The results of the evaluation will produce a new method based on Rabin-Karp method. This algorithm looks for sequence patterns in DNA [6].

\section{A.Improved Rabin-Karp Implementation}

The following table is an example of the development of the Rabin-Karp algorithm for DNA.

Table 1. Rabin-Karp DNA Test

\begin{tabular}{|c|c|c|c|c|c|c|c|}
\hline A & B & B & C & A & B & C & A \\
\hline \multicolumn{8}{|c|}{ hash $(A B B)=0$} \\
\hline \multicolumn{8}{|c|}{ q_t0 $=328965$} \\
\hline A & B & B & C & A & B & C & A \\
\hline \multicolumn{8}{|c|}{ hash $(B B C)=1$} \\
\hline \multicolumn{8}{|c|}{ q_t1 = 334026} \\
\hline A & B & B & C & A & B & C & $A$ \\
\hline \multicolumn{8}{|c|}{$\operatorname{hash}(B C A)=0$} \\
\hline \multicolumn{8}{|c|}{ q_t2 $=334045$} \\
\hline A & B & B & C & A & B & C & A \\
\hline \multicolumn{8}{|c|}{ hash $(C A B)=7$} \\
\hline \multicolumn{8}{|c|}{ q_t3 = 339047} \\
\hline A & B & $\mathrm{B}$ & C & A & B & C & $A$ \\
\hline \multicolumn{8}{|c|}{ hash $(A B C)=$} \\
\hline \multicolumn{8}{|c|}{ q_t1 = 328984} \\
\hline A & B & B & C & A & B & C & A \\
\hline \multicolumn{8}{|c|}{ hash $(B C A)=0$} \\
\hline & 33 & & & & & & \\
\hline
\end{tabular}

Table 1 describes the hash $=0$ and quotient $=334045$, both matched. BCA pattern is suitable. $\mathrm{ABB}=0$ and quotient $=328965$, which has not matched, $\mathrm{ABB}$ is not compared.

In certain cases, the development of this algorithm does not have some striking advantages with the Rabin-Karp algorithm. The complexity of this algorithm can be increased significantly [5]. Time complexity is $O((n-m+1) m)$ to $O(n m+1)$. It depends on the prime number being the modulo. Choosing the right prime numbers will improve good optimization [6]. 


\section{Methodology}

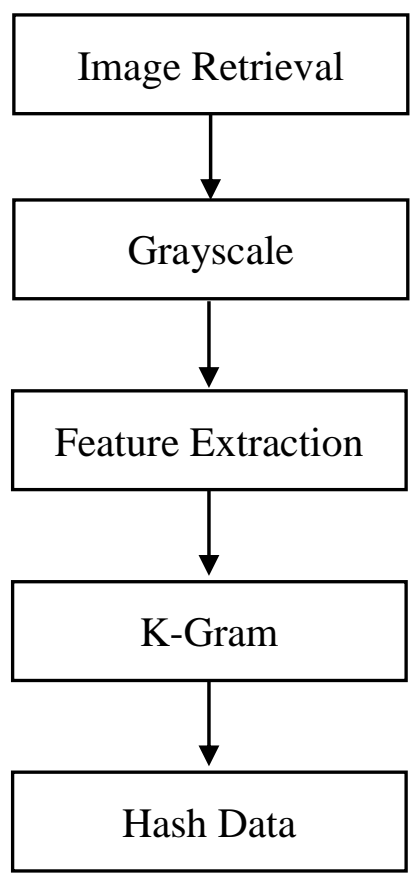

Some steps need to be taken to determine the resemblance of an image. These processes are Image Retrieval, Grayscale, Feature Extraction, K-Gram and Hash value.

\section{A. Image Retrieval}

Sampling is done to determine the object of research. The sample can be an image of a certain size. The following image is an RGB image taken from the internet that has a 300 x 300 resolution. The image must be divided into three layers, Red, Green, Blue and converted into Grayscale.

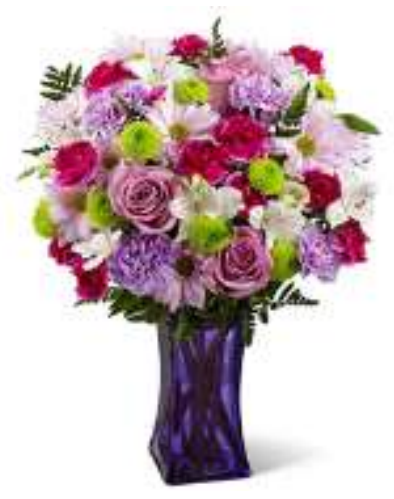

Figure 1. RGB Image

\section{B. Grayscale}

Previously specified images must be converted to grayscale mode. The method used is Luminosity. The algorithm is by multiplying each value of Red, Green, and Blue with certain constants that have been assigned value, then the result of multiplication of all values added together. The mathematical formula is:

$$
G S=(0.21 * R)+(0.72 * G)+(0.07 * B)
$$


The grayscale technique with luminosity has better brightness than the average technique. The resulting color is brighter so that pixel block determination will be better when matching the test image to the sample.

The following figure is the result of the grayscale process on the previous flower image.

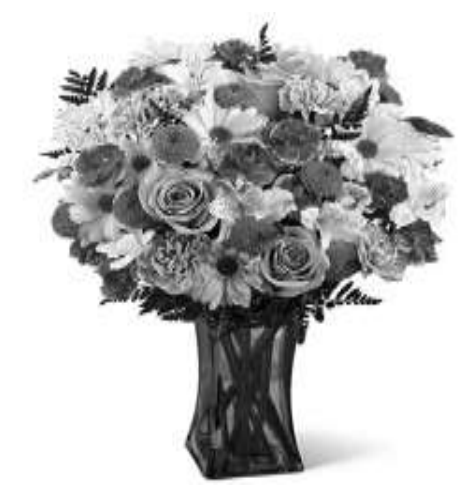

Figure 2. Grayscale Image

\section{Feature Extraction}

The coordinates used in image objects are two dimensions. This field has two coordinates $(\mathrm{M}, \mathrm{N})$. The picture below is an explanation of the image expansion in the coordinate plane.
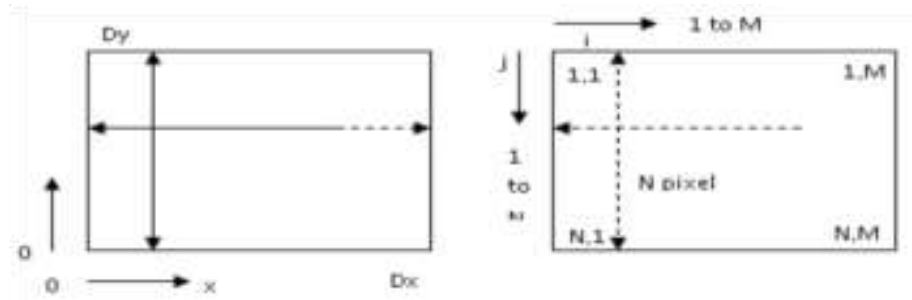

Figure 3. Coordinate Plane

This section uses a snippet of two images (Image 1 and Image 2). The image has a size of $5 \times 5$ pixels. The feature extraction on each image takes the grayscale color intensity value with the gray degree from 0 to 255 . The color intensity chunks have identical similarities. By using 25 pixels, five of them have different intensities between the two images.

Table 2. Feature Extraction Image 1

\begin{tabular}{|c|c|c|c|c|}
\hline 12 & 222 & 113 & 154 & 235 \\
\hline 248 & 252 & 227 & 108 & 169 \\
\hline 55 & 133 & 132 & 238 & 109 \\
\hline 127 & 156 & 50 & 193 & 227 \\
\hline 42 & 53 & 45 & 211 & 231 \\
\hline
\end{tabular}


Table 3. Feature Extraction Image 2

\begin{tabular}{|c|c|c|c|c|}
\hline 12 & 222 & 113 & 154 & 235 \\
\hline 248 & 250 & 227 & 108 & 169 \\
\hline 55 & 133 & 111 & 238 & 109 \\
\hline 127 & 156 & 50 & 123 & 227 \\
\hline 67 & 53 & 49 & 211 & 231 \\
\hline
\end{tabular}

Tables 2 and 3 show the results of pixel taking on grayscale images of two images. In these pictures, there are five different pixels. The percentage of pixel resemblance is $\frac{5}{25}$. This pattern has not been able to determine the level of similarity between the two images. Further calculations should be made to analyze the hash value of the image.

\section{K-Gram}

The value of K-Gram can be adjusted to the needs. This study uses the value of KGram $=5$ to determine the value of Hash in the next stage. The following table is an arrangement of K-Gram for 25 pixels.

Table 4. K-Gram

\begin{tabular}{|c|c|c|c|c|}
\hline 12 & 222 & 113 & 154 & 235 \\
\hline 222 & 113 & 154 & 235 & 248 \\
\hline 113 & 154 & 235 & 248 & 252 \\
\hline 154 & 235 & 248 & 252 & 227 \\
\hline 235 & 248 & 252 & 227 & 108 \\
\hline 248 & 252 & 227 & 108 & 169 \\
\hline 252 & 227 & 108 & 169 & 55 \\
\hline 227 & 108 & 169 & 55 & 133 \\
\hline 108 & 169 & 55 & 133 & 132 \\
\hline 169 & 55 & 133 & 132 & 238 \\
\hline 55 & 133 & 132 & 238 & 109 \\
\hline 133 & 132 & 238 & 109 & 127 \\
\hline 132 & 238 & 109 & 127 & 156 \\
\hline 238 & 109 & 127 & 156 & 50 \\
\hline 109 & 127 & 156 & 50 & 193 \\
\hline 127 & 156 & 50 & 193 & 227 \\
\hline 156 & 50 & 193 & 227 & 42 \\
\hline 50 & 193 & 227 & 42 & 53 \\
\hline 193 & 227 & 42 & 53 & 45 \\
\hline 227 & 42 & 53 & 45 & 211 \\
\hline 42 & 53 & 45 & 211 & 231 \\
\hline
\end{tabular}

\section{E. Hash Data}

Hash data is a collection of unique numbers to identify the image pattern similarity of several rows of pixels. This data aims to determine the number of pixels that are similar between the two images. The value obtained is the value to be compared between the sample image and the test image. The degree of similarity depends on the same number of hash values in both images. The following tables are the example of a hash list of two images. 
Table 5. Hash Value of Image 1

\begin{tabular}{|l|l|l|l|l|}
\hline 19875 & 16830 & 23124 & 17433 & 20546 \\
\hline 21489 & 26753 & 13498 & 23846 & 16528 \\
\hline 21848 & 28447 & 29994 & 10301 & 13009 \\
\hline 18832 & 27217 & 23157 & 25854 & 22492 \\
\hline 14952 & 14337 & 29348 & 19978 & 28809 \\
\hline 13485 & 14188 & 13131 & 21215 & 12053 \\
\hline 25669 & 13809 & 26508 & 19455 & 25356 \\
\hline 29964 & 17723 & 26633 & 17445 & 11803 \\
\hline 19477 & 27142 & 24814 & 15155 & 26266 \\
\hline 28432 & 19007 & 21896 & 16625 & 20681 \\
\hline
\end{tabular}

Table 6. Hash Value of Image 2

\begin{tabular}{|l|l|l|l|l|}
\hline 19875 & 26406 & 28424 & 13930 & 20546 \\
\hline 18049 & 10867 & 18516 & 26753 & 19975 \\
\hline 10152 & 13053 & 24120 & 21896 & 18351 \\
\hline 12605 & 25101 & 21215 & 20750 & 15513 \\
\hline 22949 & 26006 & 25045 & 25932 & 10695 \\
\hline 13254 & 21504 & 20286 & 22492 & 10615 \\
\hline 25565 & 29941 & 17403 & 23018 & 22666 \\
\hline 19744 & 19769 & 19877 & 29535 & 13139 \\
\hline 25669 & 16830 & 14297 & 20916 & 24640 \\
\hline 16960 & 20681 & 13131 & 13009 & 18947 \\
\hline
\end{tabular}

From the tables above, there are 12 same values. The next process is to calculate the percentage of similarity of the two lists. The following formula is to calculate the similarity.

Where:

$$
\mathrm{S}=\frac{2 * \mathrm{SH}}{\mathrm{TH} 1+\mathrm{TH} 2} * 100 \%
$$

$\mathrm{S}=$ Similarity Rate

$\mathrm{SH}=$ Identical Hash

TH1 = Total Hash Image 1

$\mathrm{TH} 2=$ Total Hash Image 2

The similarity rate is shown below.

$$
\begin{aligned}
\mathrm{S} & =\frac{2 * 12}{50+50} * 100 \% \\
& =\frac{24}{100} * 100 \% \\
& =0.24 * 100 \% \\
& =24 \%
\end{aligned}
$$

The percentage of similarity is $20 \%$.

\section{Experimental Result}

The test results show some significant differences. The use of different K-Gram values will trigger a change in the results of similarity levels of the image as well. The following 
table is the result of four experiments with different parameters. Three parameters are occupied in this method as the input, K-Gram, Base, and Modulo.

\begin{tabular}{lcccc} 
& Test 1 & Test 2 & Test 3 & Test 4 \\
\hline N-Gram & 5 & 3 & 6 & 2 \\
Base & 10 & 7 & 10 & 9 \\
Modulo & $\infty$ & 10007 & 3737 & 2323 \\
Image 1 & 9212 & 9214 & 9211 & 9215 \\
Image 2 & 9212 & 9214 & 9211 & 9215 \\
Identic & 4528 & 6554 & 7922 & 8576 \\
\hline Similarity & $\mathbf{4 9 , 1 5 6 \%}$ & $\mathbf{7 1 , 1 3 1 \%}$ & $\mathbf{8 6 , 0 0 6 \%}$ & $\mathbf{9 3 , 0 6 6 \%}$
\end{tabular}

The following two images show a significant but different picture. Both images are 100 $\mathrm{x} 100$ pixel. The first image is similar to the second image, but one of the images is flip horizontally. The calculation of the Rabin-Karp method will show how closely the similarities between the two images are.

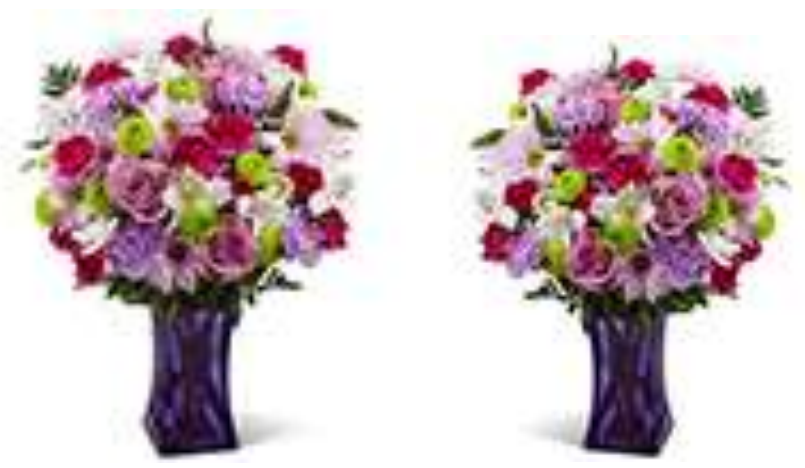

Figure 3. (a) Normal (b) Flipped

The first test is done with several input parameters, K-Gram $=5$, Base $=10$ and Modulo $=\infty$. The test results show the similarity level of $49,1553 \%$ with the same hash number is 4528 . This hash value obtained from the total hash is 18424. Each image has a hash of 9212. The second test is done with several input parameters, $\mathrm{K}-\mathrm{Gram}=3$, Base $=$ 7 and Modulo $=10007$. The test results show the similarity level of $71,1309 \%$ with the same hash number is 6554 . This hash value obtained from the total hash is 18428 . Each image has a hash of 9214.

The third test is performed with several input parameters, $\mathrm{K}-\mathrm{Gram}=6$, Base $=10$ and Modulo $=3737$. The test results show the similarity rate of $86,0059 \%$ with the same hash number is 7922. This hash value obtained from the total hash is 18422. Each image has a hash of 9211.

The last test is done with several input parameters, $\mathrm{K}-\mathrm{Gram}=2$, Base $=9$ and Modulo $=2323$. The test results show $93,0657 \%$ similarity level with the same hash number is 8576. This hash value obtained from the total hash is 18430 . Every image has a hash of 9215.

\section{Conclusion}

Rabin-Karp algorithm can be used to check image similarity based on hash value calculation. The way the calculation works is the same as that done to string matching. The fundamental difference is the feature extraction of color images. The larger the 
image, the longer the calculation process. Extraction can accelerate this process in certain parts. The grayscale process is one of the processes to speed up the image matching process. The downside of this method is a very slow process for calculating hash values in large images.

\section{Reference}

[1] S. K. Shivaji and P. S., "Plagiarism Detection by using Karp-Rabin and String Matching Algorithm Together", International Journal of Computer Applications, vol. 116, no. 23, (2015), pp. 37-41.

[2] M. Cebrián, M. Alfonseca and A. Ortega, "Towards the Validation of Plagiarism Detection Tools by Means of Grammar Evolution", IEEE Transactions on Evolutionary Computation, vol. 13, no. 3, (2009), pp. 71-77.

[3] 'A. Parker and J. O. Hamblen, "Computer Algorithm for Plagiarism Detection”, IEEE Trans. Education, vol. 32, no. 2, (1989), pp. 94-99.

[4] 'A. Apostolico, "String editing and Longest Common Subsequences", Germany: Springer-Verlag, vol. 3, (1997), pp. 1-10.

[5] Sunita, R. Malik and M. Gulia, "Rabin-Karp Algorithm with Hashing a String Matching Tool", International Journal of Advanced Research in Computer Science and Software Engineering, vol. 4, no. 3, (2014), pp. 389-392.

[6] 'A. P. Gope and R. N. Behera, "A Novel Pattern Matching Algorithm in Genome”, International Journal of Computer Science and Information Technologies, vol. 5, no. 4, (2014), pp. 5450-5457.

[7] 'A. P. U. Siahaan, Mesran, R. Rahim and D. Siregar, "K-Gram as A Determinant of Plagiarism Level in Rabin-Karp Algorithm”, International Journal of Scientific \& Technology Research, vol. 6, no. 7, (2017), pp. 350-353.

[8] R. E. Putri and A. P. U. Siahaan, "Examination of Document Similarity Using Rabin-Karp Algorithm", International Journal of Recent Trends in Engineering \& Research, vol. 3, no. 8, (2017), pp. 196-201.

[9] N. A. Putri, A. P. U. Siahaan, F. Wadly and Muslim, "Image Similarity Test Using Eigenface Calculation", International Journal of Scientific Research in Science and Technology, vol. 3, no. 6, (2017), pp. 510-515.

[10] 'A. P. U. Siahaan and R. Rahim, "Dynamic Key Matrix of Hill Cipher Using Genetic Algorithm", International Journal of Security and Its Applications, vol. 10, no. 8, (2016), pp. 173-180.

[11] 'A. P. U. Siahaan, "Vernam Conjugated Manipulation of Bit-plane Complexity Segmentation", International Journal of Security and Its Applications, vol. 11, no. 9, (2017), pp. 1-12.

[12] R. Rahim, "128 Bit Hash of Variable Length in Short Message Service Security," International Journal of Security and Its Applications, vol. 11, no. 1, (2017), pp. 45-58.

\section{Author}

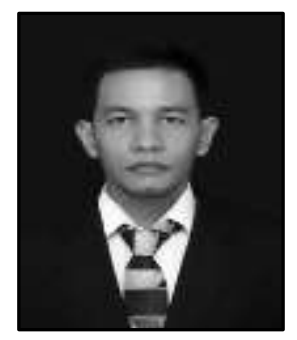

Andysah Putera Utama Siahaan, he was born in 1980, Medan, Indonesia. He received the S.Kom. degree in computer science from Universitas Pembangunan Panca Budi, Medan, Indonesia, in 2010, and in 2012, he obtained Master Degree (M.Kom.) from the University of Sumatera Utara, Medan, Indonesia. In 2010, he joined as a lecturer at the Department of Engineering, Universitas Pembangunan Panca Budi. He has been a researcher since 2012. He has studied his Ph. D. degree from 2016. He is now active in writing international journals and conferences. 